\title{
Characterizing the Cortical Activity through Which Pain Emerges from Nociception
}

\author{
Michael C. Lee, ${ }^{1,2 *}$ André Mouraux, ${ }^{3 *}$ and Gian Domenico Iannetti ${ }^{2}$ \\ ${ }^{1}$ Department of Anaesthesia, University of Cambridge, Cambridge CB2 2QQ, United Kingdom, ${ }^{2}$ Department of Physiology, Anatomy and Genetics, \\ University of Oxford, Oxford OX1 3QX, United Kingdom, and ${ }^{3}$ Centre for Functional Magnetic Resonance Imaging of the Brain, University of Oxford, \\ Oxford OX3 9DU, United Kingdom
}

Nociception begins when $\mathrm{A} \delta$ - and C-nociceptors are activated. However, the processing of nociceptive input by the cortex is required before pain can be consciously experienced from nociception. To characterize the cortical activity related to the emergence of this experience, we recorded, in humans, laser-evoked potentials elicited by physically identical nociceptive stimuli that were either perceived or unperceived. Infrared laser pulses, which selectively activate skin nociceptors, were delivered to the hand dorsum either as a pair of rapidly succeeding and spatially displaced stimuli (two-thirds of trials) or as a single stimulus (one-third of trials). After each trial, subjects reported whether one or two distinct painful pinprick sensations, associated with A $\delta$-nociceptor activation, had been perceived. The psychophysical feedback after each pair of stimuli was used to adjust the interstimulus interval (ISI) of the subsequent pair: when a single percept was reported, ISI was increased by $40 \mathrm{~ms}$; when two distinct percepts were reported, ISI was decreased by $40 \mathrm{~ms}$. This adaptive algorithm ensured that the probability of perceiving the second stimulus of the pair tended toward 0.5 . We found that the magnitude of the early-latency N1 wave was similar between perceived and unperceived stimuli, whereas the magnitudes of the later N2 and P2 waves were reduced when stimuli were unperceived. These findings suggest that the N1 wave represents an early stage of sensory processing related to the ascending nociceptive input, whereas the $\mathrm{N} 2$ and $\mathrm{P} 2$ waves represent a later stage of processing related, directly or indirectly, to the perceptual outcome of this nociceptive input.

\section{Introduction}

Nociception is defined as the neural process of encoding and processing noxious stimuli (Loeser and Treede, 2008). Although nociception is most often the cause of pain, it is not synonymous with pain, which is a conscious experience that can occur in the absence of nociception. It is now universally accepted that cortical activity is necessary for the generation of a painful experience (Bushnell and Apkarian, 2005). The cortical regions activated by nociceptive input are well described, particularly those that are consistently activated during pain (for example, the anterior insula) (Craig, 2009). However, the cortical activity specifically related to the conscious detection of nociceptive stimuli is widely undefined. Isolating such activity would thus represent a fundamental advance toward understanding the specific cortical processes through which pain emerges from nociception.

Brief radiant heat pulses, generated by infrared laser stimulators, may be used to excite selectively $\mathrm{A} \delta$ - and C-fiber free nerve endings located in the superficial layers of the skin (Bromm and Treede, 1984). Such stimuli elicit a number of electrical brain

Received Dec. 30, 2008; revised May 1, 2009; accepted May 9, 2009.

M.C.L. was supported by the United Kingdom Medical Research Council. A.M. is a Marie Curie Postdoctoral Research Fellow and a "Chargé de Recherche" of the Belgian National Fund for Scientific Research. G.D.I. is University Research Fellow of The Royal Society.

${ }^{*} M$.C.L. and A.M. contributed equally to this work.

Correspondence should be addressed to Dr. Gian Domenico lannetti, Department of Physiology, Anatomy and Genetics, University of Oxford, South Parks Road, Oxford 0X1 3QX, UK. E-mail: giandomenico.iannetti@dpag.ox.ac.uk. DOl:10.1523/JNEUROSC1.0014-09.2009

Copyright $\odot 2009$ Society for Neuroscience $\quad 0270-6474 / 09 / 297909-08 \$ 15.00 / 0$ responses, some of which can be detected in the human EEG (Carmon et al., 1976; Mouraux et al., 2003). Although the laser stimulus coactivates several distinct ascending somatosensory pathways (Iannetti et al., 2003), the detected responses have been shown to be exclusively related to the activation of type-II A $\delta$ mechano-heat nociceptors (Treede et al., 1995) and spinothalamic neurons located in the anterolateral quadrant of the spinal cord (Treede, 2003). Laser-evoked potentials (LEPs) comprise a number of waves that are time locked to the onset of the stimulus. The largest wave is a negative-positive complex maximal at the scalp vertex (N2-P2; peaking at 200-350 ms when stimulating the hand dorsum) (Bromm and Treede, 1984). This complex is preceded by a smaller negative wave $(\mathrm{N} 1$; peaking at $\sim 160 \mathrm{~ms})$ maximal over the temporal region contralateral to the stimulated side (García-Larrea et al., 1997). Several studies have shown that these waves reflect a combination of cortical activities originating from primary and secondary somatosensory cortices, the insula, and the anterior cingulate cortex (García-Larrea et al., 2003).

Here, we devised a novel experimental paradigm to determine how the N1, N2, and P2 waves of the LEP relate to the perception of pain (see Fig. 1). The experiment relied on the premise that the interval between two identical sensory stimuli is an important determinant of whether or not the second stimulus is perceived: if two stimuli are delivered close in space using a short interstimulus interval (ISI), subjects are likely to perceive them as a single percept, whereas if two stimuli are delivered using a long ISI, subjects are likely to perceive them as two distinct percepts (Mouraux et al., 2004). Indeed, it is generally accepted that spatial 


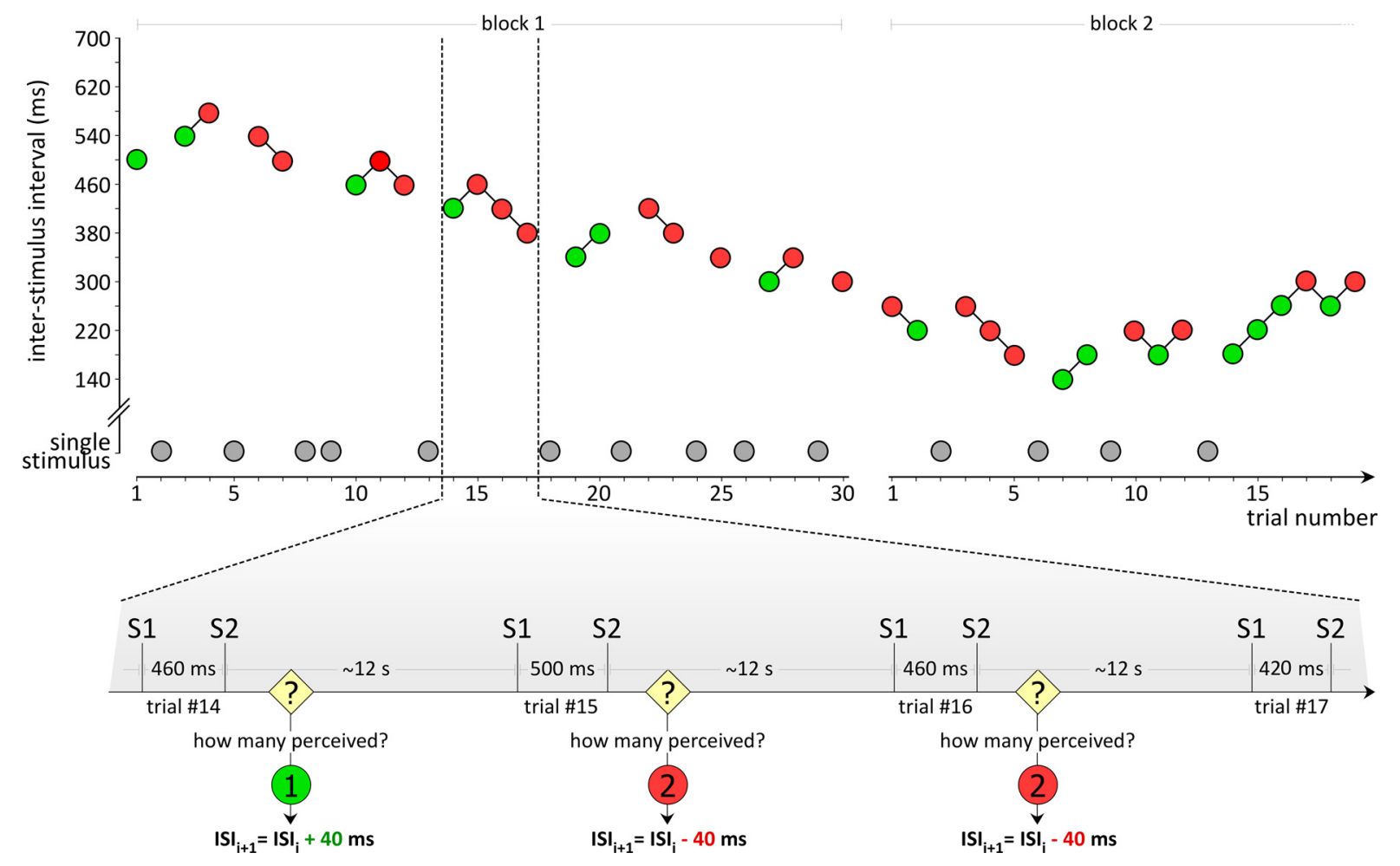

Figure 1. Experimental design. Top, Nociceptive stimuli were presented in blocks. Each block (30 trials) consisted of 20 trials during which two stimuli were applied close in space and in rapid temporal succession (green and red circles) and 10 trials during which a single stimulus was applied (gray circles). $x$-Axis, Trial number; $y$-axis, ISI. Bottom, After each trial, the subject was asked whether one or two temporally distinct pricking sensations had been perceived. The ISI of each double-stimulus trial was determined by the number of pricking sensations perceived in the preceding double-stimulus trial. If a single pricking sensation had been perceived (green), the ISl of the following double-stimulus trial was increased by $40 \mathrm{~ms}$, thus making the detection of the second stimulus easier. If two pricking sensations had been perceived (red), the ISI of the following double-stimulus trial was reduced by $40 \mathrm{~ms}$, thus making the detection of the second stimulus harder. This adaptive staircase algorithm ensured that the ISI of double-stimulus trials was maintained, throughout the experiment, at a value at which the likelihood of perceiving the second stimulus was equal to the likelihood of not perceiving the second stimulus.

proximity and temporal simultaneity are two main factors that determine whether physically separated stimuli are integrated as a unified percept (Meredith et al., 1987; King and Calvert, 2001). Here, we characterized the relationship between conscious perception and the magnitude of the N1, N2, and P2 waves by comparing the LEPs elicited by perceived and unperceived stimuli.

\section{Materials and Methods}

Subjects. Ten healthy, right-handed subjects participated in the study (four males and six females; aged between 24 and 38 years). Subjects were recruited from research staff and students at the University of Oxford (Oxford, UK). All participants gave written informed consent. The Oxfordshire Local Research Ethics Committee approved the study (REC: 07/Q1603/9).

Nociceptive stimulation. Pulses of radiant heat were generated by an infrared neodymium:yttrium-aluminum-perovskite (Nd:YAP) laser with a wavelength of $1.34 \mu \mathrm{m}$ (Electronical Engineering). The laser pulse was transmitted via an optic fiber and focused by lenses to a spot diameter of $\sim 8 \mathrm{~mm}$ at the target site. After each stimulus, the spot location was shifted by $20 \mathrm{~mm}$ in a random direction, to avoid nociceptor fatigue and sensitization. The spot location was controlled by a computer that used two servo-motors (HS-422; Hitec RCD; angular speed, $60 \% 160 \mathrm{~ms}$ ) to orient the laser beam along two perpendicular axes (see supplemental video, available at www.jneurosci.org as supplemental material). Each spot location was assigned a coordinate on a two-dimensional spatial grid (proximal-distal axis, $\sim 4 \mathrm{~cm}$; medial-lateral axis, $\sim 4 \mathrm{~cm}$ ) whose limits were adjusted to fit within the left-hand dorsum (see Fig. 2).

To familiarize subjects with the nociceptive stimulus, a small number of low-energy laser pulses were delivered to the left-hand dorsum. The energy of the stimulus was then adjusted to elicit a clear pricking pain sensation $(2.4 \pm 0.2 \mathrm{~J})$, related to the activation of $\mathrm{A} \delta$-nociceptors (Treede et al., 1995).

Experimental design. In a previous study we showed that if two identical laser stimuli are applied close in space (i.e., $\sim 2 \mathrm{~cm}$ ) and time (i.e., ISI $<600 \mathrm{~ms}$ ), the likelihood of perceiving the second stimulus as a distinct percept is strongly reduced (Mouraux et al., 2004). In the present experiment, we took advantage of this psychophysical phenomenon to compare the brain responses elicited by a pair of physically identical laser stimuli, the second of which was either perceived or not perceived. For each participant, the ISI was adjusted continuously throughout the experiment, such that the likelihood of perceiving or not perceiving the second stimulus as a distinct percept tended to be equal. The experimental paradigm we used bears some resemblance to the attentional blink paradigm, in which two visual targets are presented within a continuous flow of nontarget stimuli (Raymond et al., 1992). Studies using such paradigms have shown that, when the time interval between the two target stimuli is short (typically $<500 \mathrm{~ms}$ ) and when the second target stimulus is quickly masked by a subsequent stimulus, the ability to detect the second target is significantly impaired (Giesbrecht and Di Lollo, 1998). This attentional blink phenomenon is thought to result from the early cortical representation of the second target being overwritten by the subsequent nontarget stimulus, while the central processes required for its consolidation are still engaged by the first target. In the present experiment, because the second stimulus of the pair was not followed by a subsequent masking stimulus, we did not expect to observe such a phenomenon, although both the paradigm we used and the attentional blink paradigm aim to identify neural activity related to the conscious detection of a sensory stimulus.

The experiment consisted of five blocks (Fig. 1). Each block was separated by a 5 min interval and consisted of 20 trials in which two stimuli 
Table 1. Behavioral performance

\begin{tabular}{|c|c|c|}
\hline Subject & $\begin{array}{l}\text { Mean ( } \pm \text { SD) ISI of double-stimulus } \\
\text { trials (ms) }\end{array}$ & $\begin{array}{l}\text { Single-stimulus trials perceived as } \\
\text { double stimuli (\%) }\end{array}$ \\
\hline 1 & $297 \pm 176$ & 30 \\
\hline 2 & $548 \pm 111$ & 0 \\
\hline 3 & $341 \pm 36$ & 8 \\
\hline 4 & $375 \pm 49$ & 22 \\
\hline 5 & $780 \pm 121$ & 4 \\
\hline 6 & $904 \pm 75$ & 5 \\
\hline 7 & $238 \pm 166$ & 48 \\
\hline 8 & $1096 \pm 382$ & 10 \\
\hline 9 & $395 \pm 121$ & 16 \\
\hline 10 & $558 \pm 239$ & 30 \\
\hline Mean $\pm S D$ & $553 \pm 286$ & $17 \pm 15$ \\
\hline
\end{tabular}

were applied in rapid succession and 10 trials in which a single stimulus was applied. The intertrial interval was $10-15$ s. Double-stimulus and single-stimulus trials were intermingled randomly. After each trial, the subject was asked to report whether one or two temporally distinct pricking sensations had been perceived. This specific instruction was required because the laser stimulus concomitantly activates $\mathrm{A} \delta$ - and C-nociceptors, thus eliciting a dual sensation of "first pain" and "second pain" (Lewis and Ponchin, 1937). First pain, related to the activation of A $\delta$-nociceptors, is characterized by a distinct pricking quality. Second pain, related to the activation of C-nociceptors, is characterized by a diffuse, long-lasting warm or burning sensation (Nahra and Plaghki, 2003). By asking subjects to detect a stimulus-triggered "pricking" sensation, we thus made sure that the sensation of second pain elicited by the first stimulus was not interfering with the detection of the first pain sensation elicited by the second stimulus. The ISI for the first doublestimulus trial was $500 \mathrm{~ms}$. The ISI of the following double-stimulus trial was determined by the number of percepts elicited by the preceding double-stimulus trial: if a single pricking sensation was perceived (P1), the ISI was increased by $40 \mathrm{~ms}$. If two pricking sensations were perceived (P2), the ISI was reduced by $40 \mathrm{~ms}$.

This adaptive staircase algorithm ensured that the ISI of doublestimulus trials was maintained, throughout the experiment, at a value at which the likelihood of perceiving and not perceiving the second stimulus was equal. Importantly, the physical characteristics of both the first and the second laser stimulus were identical throughout the experiment. Hence, the likelihood of perceiving or not perceiving the second laser stimulus was unlikely to depend on differences in the incoming nociceptive input. The inclusion of single-stimulus trials reduced the effect of expectation on the EEG responses elicited by the second stimulus. Because single-stimulus trials were included, the overall probability of perceiving a second stimulus tended toward $p=0.33$. Also, the number of percepts reported after a single-stimulus trial was used to assess whether the subjective reports of perception during double-stimulus trials were reliable (Table 1).

Control experiment. To explore the intensity of the perceived sensation, we performed an additional experiment on six healthy, righthanded subjects (four males and two females; aged between 24 and 35 years). The same adaptive stimulation paradigm used during the LEP experiment was used for the control experiment (Fig. 1). Sixty trials (two blocks of 30 trials: 10 single-stimulus trials and 20 double-stimulus trials) were delivered to the dorsum of the right hand. After each trial, subjects were asked to report the number of perceived pricking sensations, as well as the intensity of each elicited pricking pain percept, using a numerical rating scale ranging from 0 to 10 , where 0 was defined as "no pain" and 10 was defined as "pain as bad as it could be" (Jensen et al., 1989). The intensity ratings given by each subject was rescaled so that 0 and 100 represented the lowest and highest pain rating of every subject.

EEG recording. All subjects were seated in a comfortable chair and wore protective goggles. They were asked to focus on the sensations that arose from hand stimulation, relax, and keep their eyes open and gaze slightly downward. Acoustic isolation was ensured using headphones through which white noise was played at comfortable listening level.
The EEG was recorded using $30 \mathrm{Ag}-\mathrm{AgCl}$ electrodes placed on the scalp according to the International 10-20 system, using the nose as common extracephalic reference. The electrooculogram was recorded using two electrodes; one placed at the upper left and the other at the lower right side of the right eye. The electrocardiogram was recorded using two electrodes placed at the left and right wrist. Signals were amplified and digitized using a sampling rate of $1024 \mathrm{~Hz}$ and a precision of 12 bits, giving a resolution of $0.195 \mu \mathrm{V}$ (SD32; Micromed).

EEG analysis. Analyses were performed using Letswave (http:// amouraux.webnode.com) (Mouraux and Iannetti, 2008a), EEGLAB (http://sccn.ucsd.edu), and Matlab (Mathworks).

Continuous EEG recordings were segmented into epochs, -1 to $2 \mathrm{~s}$ relative to the onset of all stimuli. After off-line bandpass filtering (0.5-40 Hz fast Fourier transform filter), electrooculographic and electrocardiographic artifacts were removed using a validated method based on an independent component (IC) analysis (Jung et al., 2000). In all datasets, ICs related to eye movements had a large electrooculogram channel contribution and a frontal scalp distribution. The number of ICs removed from each dataset ranged from 2 to 4 . Epochs were then baseline-corrected (reference interval, -1 to $0 \mathrm{~s}$ ). Finally, epochs containing artifacts exceeding $\pm 100 \mu \mathrm{V}$ were rejected from additional analysis.

The spatial distributions of the stimulus location of unperceived (P1) and perceived (P2) double-stimulus trials were defined by the frequencies of trials occurring within bins of equal areas arranged in a $4 \times 4$ grid along the proximal-distal and medial-lateral axes of the hand dorsum (Fig. 2, left). An iterative procedure was then used to successively remove trials from the pools of $\mathrm{P} 1$ and $\mathrm{P} 2$ trials, until the relative frequency of trials did not differ by $>5 \%$ in each bin (Fig. 2, right). A similar procedure was then performed to match the distribution of ISI across P1 and P2 double-stimulus trials. On average, this trial selection procedure led to the removal of $13 \pm 4 \%$ of trials.

Finally, average waveforms were computed, time-locked to the onset of the first (S1) and the second (S2) stimulus of P1 and P2 doublestimulus trials, thus yielding four average waveforms for each subject: $\mathrm{S} 1 / \mathrm{P} 1, \mathrm{~S} 1 / \mathrm{P} 2, \mathrm{~S} 2 / \mathrm{P} 1$, and S2/P2. For each waveform, the latency and the baseline-to-peak amplitude of N1, N2, and P2 waves were measured as follows. The N1 wave was measured at the temporal electrode contralateral to the stimulated side (T4), referenced to Fz. It was defined as the negative deflection preceding the N2 wave, which appears as a positive deflection in this montage. The N2 and P2 waves were measured at the vertex $(\mathrm{Cz})$ referenced to the nose. The N2 wave was defined as the most negative deflection after stimulus onset. The P2 wave was defined as the most positive deflection after stimulus onset.

Statistical analyses. Statistical comparisons were performed using Matlab (Mathworks).

A two-way repeated-measures ANOVA was used to examine the main factors of "perception" (P1, second stimulus not perceived, vs P2, second stimulus perceived) and stimulus "repetition" ( $\mathrm{S} 1$, first stimulus, vs S2, second stimulus), as well as the possible interaction between the two factors, on the amplitudes and latencies of the N1, N2, and P2 waves. When significant, post hoc paired $t$ tests were used to perform pairwise comparisons.

\section{Results}

All subjects reported a clear painful pricking sensation to laser stimulation. The mean intensities of the first percept elicited by single- and double-stimulus trials and of the second percept elicited by double-stimulus trials were not significantly different $(F=0.88 ; p=0.527)$ (supplemental Fig. S1, available at www. jneurosci.org as supplemental material). Table 1 shows the mean ( \pm SD) ISI for all double-stimulus trials, as well as the percentage of single-stimulus trials that led to the perception of two stimuli. All stimuli elicited a negative wave (N2) followed by a positive wave (P2), both maximal at the vertex (Fig. 3). This complex was preceded by a smaller negative wave (N1) maximal over the temporal region contralateral to the stimulated side (Figs. 4, 5). 


\section{N1 wave}

The N1 wave was clearly identified in the average waveforms of 9 of 10 subjects.

The mean $( \pm$ SD) baseline-to-peak amplitude of the N1 wave elicited by the first stimulus of the pair (S1) was $-2.7 \pm$ $1.3 \mu \mathrm{V}$ when the second stimulus was not perceived $(\mathrm{S} 1 / \mathrm{P} 1)$ and $-3.5 \pm 2.7 \mu \mathrm{V}$ when it was perceived $(\mathrm{S} 1 / \mathrm{P} 2)$. The mean baseline-to-peak amplitude of the N1 wave elicited by the second stimulus of the pair (S2) was $-1.5 \pm 1.0 \mu \mathrm{V}$ when the second stimulus was not perceived (S2/P1) and $-1.9 \pm 2.1 \mu \mathrm{V}$ when it was perceived (S2/P2). There was no significant effect of the factor perception on the magnitude of the $\mathrm{N} 1$ waves elicited by the first and the second stimulus $(p=0.368)$, and there was no significant interaction between the effects of the factors perception and repetition ( $p=0.350$ ) (Table 2; Fig. 4, bottom left). Thus, the N1 wave elicited by the first and the second stimulus was not affected by whether or not the second stimulus was perceived. In contrast, the magnitude of the N1 wave was significantly affected by the factor repetition ( $p=0.004)$ (Table 2; Fig. 5, bottom left): the N1 wave elicited by the second stimulus was significantly smaller than the N1 wave elicited by the first stimulus, independently of whether the second stimulus was perceived or not perceived (Table 3 ).

The mean peak latency of the N1 wave was $173 \pm 21 \mathrm{~ms}$ for S1/P1, $168 \pm 24 \mathrm{~ms}$ for $\mathrm{S} 1 / \mathrm{P} 2,170 \pm 23 \mathrm{~ms}$ for $\mathrm{S} 2 / \mathrm{P} 1$, and $170 \pm 21 \mathrm{~ms}$ for $\mathrm{S} 2 / \mathrm{P} 2$. There were no significant main effects of the factors perception and repetition on the latency of the N1 waves, and there was no significant interaction between the factors perception and repetition.

\section{N2 wave}

The N2 wave was clearly identified in the average waveform of all subjects (Fig. 3 ).

The mean baseline-to-peak amplitude of the N2 wave elicited by the first stimulus of the pair (S1) was $-9.9 \pm 4.3 \mu \mathrm{V}$ when the second stimulus was not perceived (S1/ $\mathrm{P} 1)$ and $-10.8 \pm 5.1 \mu \mathrm{V}$ when the second stimulus was perceived (S1/P2). The mean baseline-to-peak amplitude of the N2 wave elicited by the second stimulus of the pair (S2) was $-4.8 \pm 2.1 \mu \mathrm{V}$ when the second stimulus was not perceived (S2/P1) and $-9.5 \pm 4.7 \mu \mathrm{V}$ when the second stimulus was perceived (S2/P2). There was a significant main effect of the factor perception on the magnitude of the N2 waves elicited by the first or the second stimulus $(p=0.015)$, and there was a significant interaction between the factors perception and repetition ( $p=0.030$ ) (Table 2; Fig. 4, top left). Post hoc comparisons revealed that, when the second stimulus was perceived, the magnitude of the N2 wave elicited by the second stimulus was en-

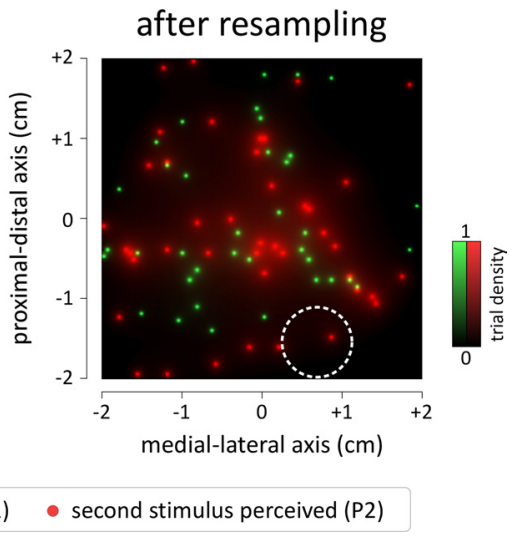

Figure 2. Spatial distribution of laser stimuli. Left, Target location of the second stimulus in trials in which the second stimulus was not perceived (red) and was perceived (green). Each spot location was assigned a pair of relative coordinates on a $4 \times 4 \mathrm{~cm}$ plane, whose area was adjusted to fit within the left-hand dorsum. Data from a representative subject are shown. Right, To ensure that differences between perceived and unperceived trials did not result from differences in peripheral nociceptive input, an iterative trial selection procedure was used to successively remove trials from the pools of perceived and unperceived trials, until their spatial distribution was homogeneous (see also Materials and Methods). Note how, in this subject, the procedure rejected a small, spatially isolated cluster of trials in which the second stimulus was not perceived (dashed circle).

First stimulus (S1)

Second stimulus (S2)
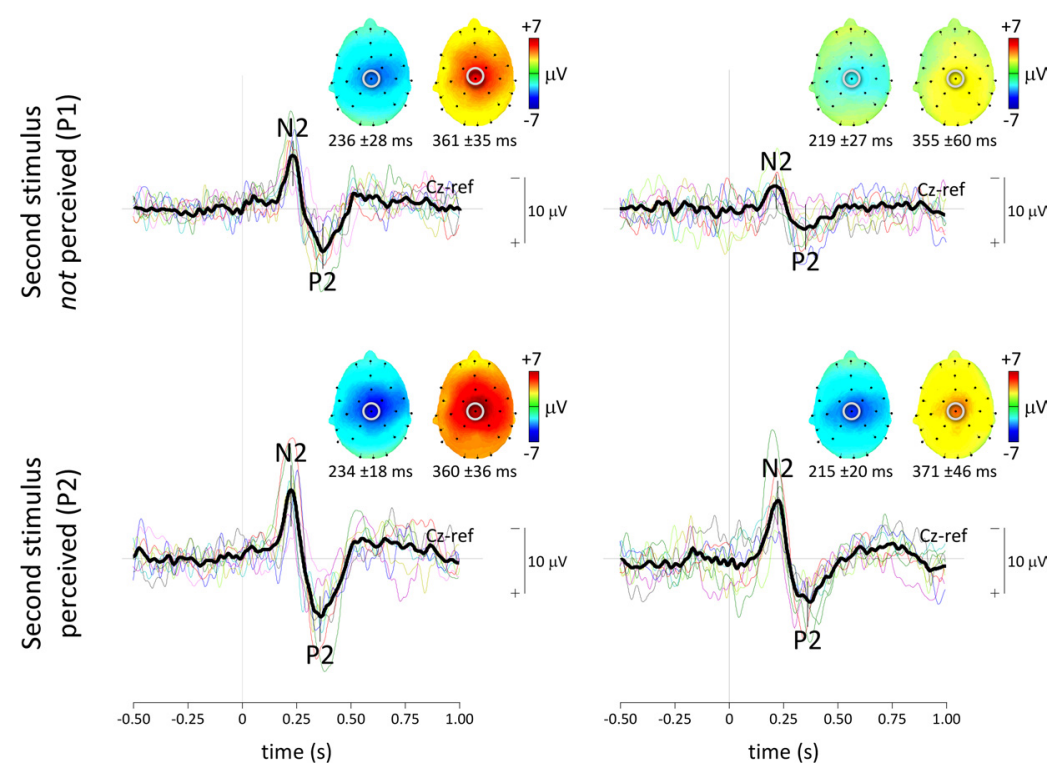

Figure 3. Time course and scalp topography of LEPs elicited by two rapidly succeeding laser stimuli. Left column, LEPs elicited by the first stimulus (S1). Right column, LEPs elicited by the second stimulus (S2). Top row, LEPs obtained when the second stimulus was not perceived (P1). Bottom row, LEPs obtained when the second stimulus was perceived (P2). The average interstimulus interval was $553 \pm 285 \mathrm{~ms}$. The colored waveforms represent single subjects, whereas the black waveforms represent the group-level average ( $\mathrm{z}$ vs nose reference). $x$-Axis, Time (in seconds). The vertical calibration bar represents amplitude (10 $\mu \mathrm{V}$; negativity plotted upward). All stimuli elicited a negative wave (N2) followed by a positive wave (P2), maximal at Cz. The topographies of both waves are displayed in the corresponding scalp maps. The gray circles mark the position of electrode $\mathrm{z}$. Note that the magnitudes of the $\mathrm{N} 2$ and $\mathrm{P} 2$ waves elicited by the second stimulus were greater when the second stimulus was perceived. Also note that the magnitudes of $\mathrm{N} 2$ and $\mathrm{P} 2$ waves elicited by the first stimulus were greater than those elicited by the second stimulus.

hanced, whereas the magnitude of the N2 wave elicited by the first stimulus was unchanged (Table 3 ). Furthermore, there was a significant main effect of the factor repetition on the magnitude of the N2 wave ( $p=0.001)$ (Table 2; Fig. 5, top left): post hoc comparisons revealed that the $\mathrm{N} 2$ wave elicited by the second stimulus was significantly smaller than the N2 wave elicited by the first stimulus when the second stimulus was not perceived, but not when the second stimulus was perceived (Table 3 ).

The mean peak latency of the N2 wave was $236 \pm 28 \mathrm{~ms}$ for 


\section{Effect of factor 'PERCEPTION'}
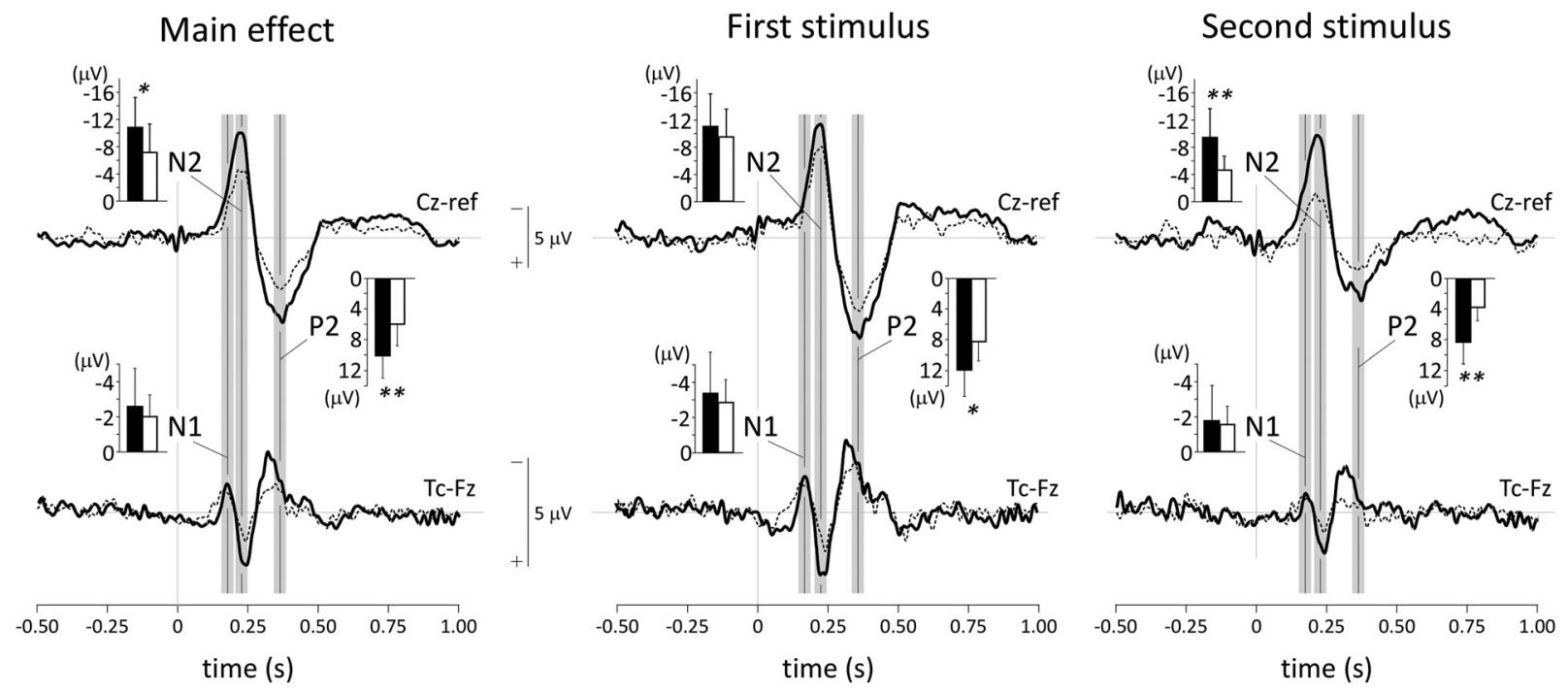

$\square$-........ second stimulus not perceived (P1)

- second stimulus perceived (P2)

Figure 4. Effect of stimulus perception on LEPs elicited by two rapidly succeeding stimuli. $x$-Axis, Time (in seconds). The vertical calibration bar represents amplitude ( $5 \mu \mathrm{V}$; negativity plotted upward). Top graphs, N2 and P2 waves recorded at the vertex (Cz vs nose reference). Bottom graphs, N1 wave recorded at the temporal region contralateral to the stimulated side (Tc vs Fz). Full waveforms, LEPs obtained when the second stimulus was perceived. Dashed waveforms, LEPs obtained when the second stimulus was not perceived. The main effect of perception is shown in the left column. Note that the magnitudes of the N2 and P2 waves were significantly greater when the second stimulus was perceived, whereas the magnitude of the N1 wave was not significantly affected by whether or not the stimulus was perceived. The effect of perception on the LEPs elicited by the first stimulus is shown in the middle column, and its effect on the LEPs elicited by the second stimulus is shown in the right column. Note how the amplitude of the P2 wave elicited by both the first and the second stimulus was significantly greater when the second stimulus was perceived. The bar graphs represent the average ( \pm SD) amplitudes of $\mathrm{N} 1, \mathrm{~N} 2$, and $\mathrm{P} 2$ waves in each condition. ${ }^{*} p<0.05 ;{ }^{* *} p<0.01$.

$\mathrm{S} 1 / \mathrm{P} 1,234 \pm 18 \mathrm{~ms}$ for $\mathrm{S} 1 / \mathrm{P} 2,219 \pm 27 \mathrm{~ms}$ for S2/P1, and $215 \pm$ $20 \mathrm{~ms}$ for S2/P2. There was no significant main effect of the factors perception and repetition on the latency of the $\mathrm{N} 2$ waves, and there was no significant interaction between the factors perception and repetition.

\section{P2 wave}

The P2 wave was identified in the average waveform of all subjects (Fig. 3).

The mean baseline-to-peak amplitude of the P2 wave elicited by the first stimulus of the pair (S1) was $8.6 \pm 2.7 \mu \mathrm{V}$ when the second stimulus was not perceived (S1/P1) and $11.8 \pm 3.7 \mu \mathrm{V}$ when the second stimulus was perceived (S1/P2). The mean baseline-to-peak amplitude of the $\mathrm{P} 2$ wave elicited by the second stimulus of the pair (S2) was $3.9 \pm 2.0 \mu \mathrm{V}$ when the second stimulus was not perceived $(\mathrm{S} 2 / \mathrm{P} 1)$ and $8.3 \pm 3.1 \mu \mathrm{V}$ when the second stimulus was perceived (S2/P2). There was a significant main effect of the factor perception on the magnitude of the P2 waves elicited by the first or the second stimulus $(p=0.002)$, but there was no significant interaction between the factors perception and repetition ( $p=0.362$ ) (Table 2; Fig. 4, top left). Thus, the P2 waves elicited by S1 and S2 were similarly affected by perception: when the second stimulus was perceived, both the magnitude of the P2 wave elicited by the first stimulus and the magnitude of the P2 wave elicited by the second stimulus were enhanced (Table 3). Furthermore, there was a significant main effect of the factor repetition on the magnitude of the P2 wave $(p=0.005)$ (Table 2; Fig. 5, top left): post hoc comparisons revealed that the $\mathrm{P} 2$ wave elicited by the second stimulus was significantly smaller than the P2 wave elicited by the first stimulus, independently of whether the second stimulus was perceived or not perceived (Table 3 ).
The mean peak latency of the $\mathrm{P} 2$ wave was $361 \pm 35 \mathrm{~ms}$ for $\mathrm{S} 1 / \mathrm{P} 1,360 \pm 36 \mathrm{~ms}$ for $\mathrm{S} 1 / \mathrm{P} 2,355 \pm 60 \mathrm{~ms}$ for $\mathrm{S} 2 / \mathrm{P} 1$, and $371 \pm$ $46 \mathrm{~ms}$ for $\mathrm{S} 2 / \mathrm{P} 2$. There was no significant main effect of the factors perception and repetition on the latency of the $\mathrm{P} 2$ waves, and there was no significant interaction between the factors perception and repetition.

\section{Discussion}

Nociceptive stimulation triggers a number of temporally distinct cortical processes, reflected by the N1, N2, and P2 waves of laserevoked potentials. Here, by examining how these waves relate to the conscious detection of a nociceptive stimulus, we investigated the specific cortical processes through which pain emerges from nociception.

The study yielded four main findings. (1) The magnitude and latency of the $\mathrm{N} 1$ waves elicited by perceived and unperceived stimuli were similar. This indicates that the N1 represents an early stage of sensory processing during which the perceptual outcome of nociceptive input is not yet determined. (2) The magnitudes of both the N2 and the P2 waves elicited by perceived stimuli were significantly larger than those elicited by unperceived stimuli. This indicates that the $\mathrm{N} 2$ and $\mathrm{P} 2$ represent a later stage of sensory processing that is related to the perceptual outcome of nociceptive input. (3) The magnitude of the P2 wave elicited by the first stimulus predicted the conscious detection of the second stimulus (i.e., its magnitude was significantly greater in trials in which the subsequent second stimulus was perceived). This suggests that the state of responsiveness of the cortical generators of the P2 wave influences the conscious detection of nociceptive input. (4) The magnitudes of the N1, N2, and P2 waves were all significantly reduced by stimulus repetition, regardless of whether the stimulus was perceived. This suggests that the effect of stimulus repe- 


\section{Effect of factor 'REPETITION'}
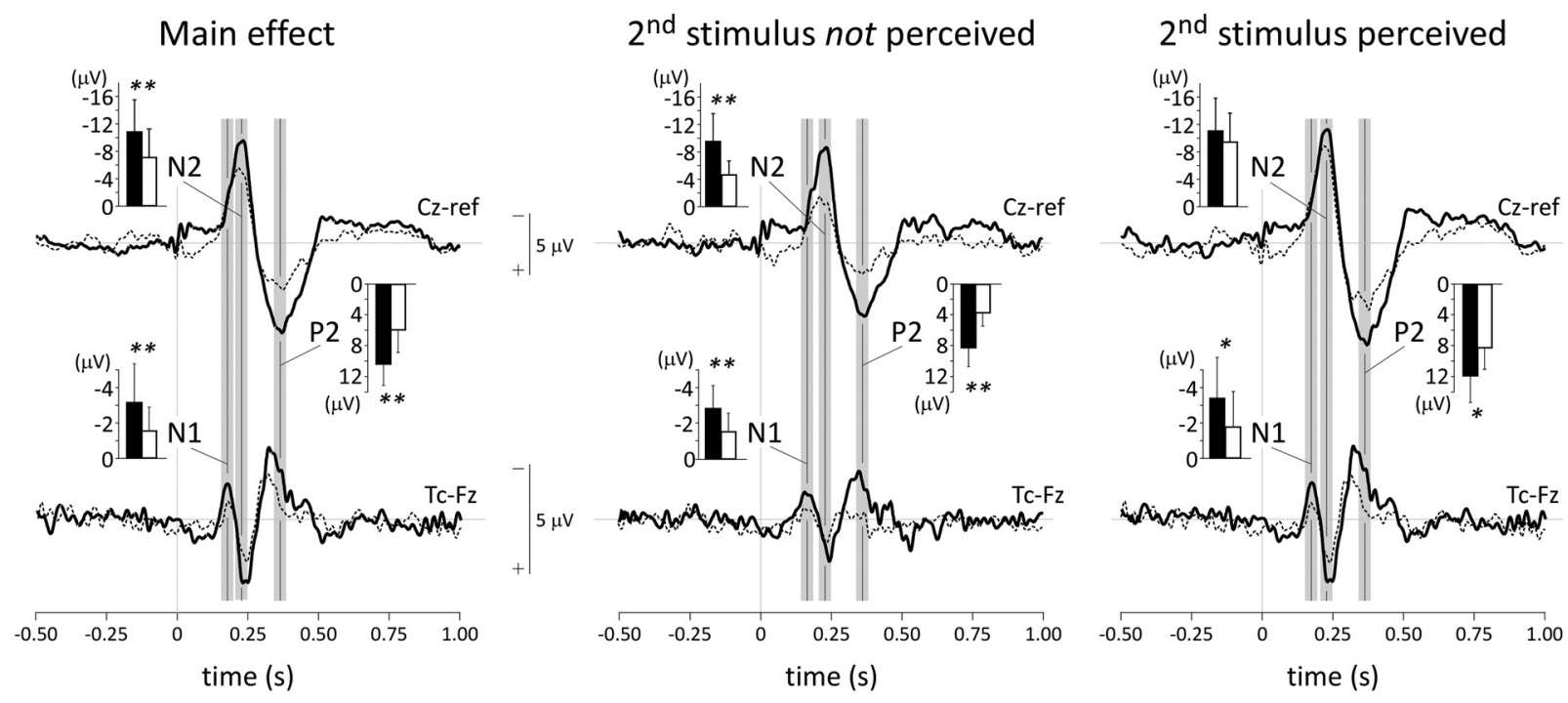

$\square$ - first stimulus (S1) $\square \cdots \cdots-$ s.... second stimulus (S2)

Figure 5. Effect of stimulus repetition on LEPs elicited by two rapidly succeeding stimuli. $x$-axis, Time (in seconds). The vertical calibration bar represents amplitude (5 $\mu V$; negativity plotted upward). Top graphs, N2 and P2 waves recorded at the vertex (Cz vs nose reference). Bottom graphs, N1 wave recorded at the temporal region contralateral to the stimulated side (Tc vs Fz). Full waveforms, LEPs elicited by the first stimulus. Dashed waveforms, LEPs elicited by the second stimulus. The main effect of stimulus repetition is shown in the left column. Note that the magnitudes of all three waves were significantly reduced by stimulus repetition. The effect of stimulus repetition on the LEPs obtained when the second stimulus was not perceived is shown in the middle column, whereas its effect on the LEPs obtained when the second stimulus was perceived is shown in the right column. The bar graphs represent the average ( \pm SD) amplitudes of N1, N2, and P2 waves in each condition. ${ }^{*} p<0.05 ;{ }^{* *} p<0.01$.

Table 2. Summary of ANOVA results

\begin{tabular}{lll}
\hline & $F$ value & $p$ value \\
\hline N1 wave & & \\
$\quad \begin{array}{l}\text { Main effect of perception } \\
\text { Main effect of repetition }\end{array}$ & $F=0.90$ & $p=0.368$ \\
Interaction between perception and repetition & $F=0.97$ & $\begin{array}{l}p=0.004^{* *} \\
p=0.350\end{array}$ \\
N2 wave & & \\
$\quad$ Main effect of perception & $F=9.43$ & $p=0.015^{*}$ \\
$\quad$ Main effect of repetition & $F=24.35$ & $p=0.001^{* *}$ \\
Interaction between perception and repetition & $F=6.93$ & $p=0.030^{*}$ \\
P2 wave & & \\
$\quad$ Main effect of perception & $F=20.92$ & $p=0.002^{* *}$ \\
Main effect of repetition & $F=14.53$ & $p=0.005^{* *}$ \\
Interaction between perception and repetition & $F=0.93$ & $p=0.362$ \\
\hline
\end{tabular}

${ }^{*} p<0.05 ;{ }^{* *} p<0.01$.

tition on nociceptive sensory processing does not require the conscious detection of the incoming sensory input.

\section{The N1 wave reflects early stages of sensory processing,} related to nociceptive input

The amplitude and latency of the $\mathrm{N} 1$ waves elicited by perceived and unperceived laser stimuli were similar (Fig. 4, bottom). This indicates that the N1 wave, which is thought to originate mainly from the operculo-insular region contralateral to the stimulated side and, possibly, from the contralateral primary somatosensory cortex (García-Larrea et al., 2003), reflects early stages of sensory processing that occur regardless of whether the nociceptive stimulus reaches conscious awareness. Since the physical characteristics of perceived and unperceived nociceptive stimuli were matched, our finding suggests that the magnitude of the $\mathrm{N} 1$ wave
Table 3. Summary of post hoct tests

\begin{tabular}{|c|c|c|c|}
\hline & N1 wave & N2 wave & P2 wave \\
\hline \multicolumn{4}{|l|}{ Effect of stimulus repetition ( $\mathrm{S} 2$ vs $\mathrm{S} 1$ ) } \\
\hline Second stimulus not perceived & $\begin{array}{l}T=4.43 \\
p=0.0016^{* *}\end{array}$ & $\begin{array}{l}T=4.84 \\
p=0.0013^{* *}\end{array}$ & $\begin{aligned} T & =4.84 \\
p & =0.0021^{* *}\end{aligned}$ \\
\hline Second stimulus perceived & $\begin{array}{l}T=3.10 \\
p=0.0127^{*}\end{array}$ & $\begin{array}{l}T=1.52 \\
p=0.1657\end{array}$ & $\begin{aligned} T & =2.48 \\
p & =0.0380^{*}\end{aligned}$ \\
\hline \multicolumn{4}{|l|}{ Effect of perception (P2 vs P1) } \\
\hline First stimulus & $\begin{array}{l}T=0.69 \\
p=0.2946\end{array}$ & $\begin{array}{l}T=1.05 \\
p=0.3260\end{array}$ & $\begin{array}{l}T=3.31 \\
p=0.0107^{*}\end{array}$ \\
\hline Second stimulus & $\begin{array}{l}T=0.69 \\
p=0.5200\end{array}$ & $\begin{array}{l}T=3.42 \\
p=0.0090^{* *}\end{array}$ & $\begin{array}{l}T=3.95 \\
p=0.0042^{* *}\end{array}$ \\
\hline
\end{tabular}

${ }^{*} p<0.05 ;{ }^{* *} p<0.01$ (corrected for multiple comparisons).

is mainly driven by the magnitude of the eliciting nociceptive input, rather than by the magnitude of pain perception. Consistent with this view, Baumgärtner et al. (2006) showed that the N1 wave can be recorded in anesthetized monkeys. Therefore, our results suggest that the correlation between the magnitude of the $\mathrm{N} 1$ wave and pain perception that was reported in two of our recent studies did not result from a relationship between the N1 wave and pain perception per se, but instead, was mostly determined by explicit (Iannetti et al., 2008) or unavoidable (Iannetti et al., 2005) variations in the strength of the peripheral nociceptive input.

Our finding is consistent with experimental observations reported in other sensory modalities. Indeed, studies examining the cortical processing of innocuous somatosensory stimuli (Libet et al., 1967; Meador et al., 2002; Schubert et al., 2006) have also shown that early cortical responses (e.g., the P60 and the N80 waves) are consistently elicited by near-threshold somatosensory stimuli regardless of whether they are perceived, whereas later 
responses (e.g., the N140 and the P250 wave) become prominent only when the stimulus is perceived.

\section{The N2 and P2 waves reflect later stages of sensory processing, related to the perceptual outcome of the nociceptive input}

In contrast to the behavior of the $\mathrm{N} 1$ wave, the magnitudes of the $\mathrm{N} 2$ and $\mathrm{P} 2$ waves elicited by perceived stimuli were significantly greater than the magnitudes of the N2 and P2 waves elicited by unperceived stimuli (Fig. 4). This indicates that the N2 and P2 waves reflect cortical processes related, directly or indirectly, to the conscious detection of nociceptive input, and agrees with the early findings by Carmon et al. (1978), showing that the magnitude of the laser-evoked N2 and P2 waves correlates better with the intensity of perception than with the intensity of the nociceptive stimulus per se.

It should be noted that unperceived stimuli were still able to elicit N2 and P2 waves, albeit of much reduced magnitude (Fig. 3) (see also the single-trial analysis reported in supplemental Fig. S2, available at www.jneurosci.org as supplemental material). This observation suggests that not all cortical activities underlying the $\mathrm{N} 2$ and P2 waves reflect neural processes related to the conscious detection of the nociceptive stimulus. In agreement with this notion, it is well established that N2 and P2 waves do not originate from a single discrete cortical area, but from an extensive array of cortical areas including bilateral operculo-insular regions and the anterior cingulate cortex (García-Larrea et al., 2003), and there is no reason to suppose that all cortical areas contributing to the N2 and P2 waves are involved in the conscious detection of the nociceptive stimulus. Some regions (for example, the anterior insula) may play a greater role in stimulus awareness (Craig, 2009).

Interestingly, the magnitude of the $\mathrm{P} 2$ wave elicited by the first stimulus was significantly greater in trials in which the second stimulus was perceived compared with trials in which the second stimulus was not perceived. In other words, the magnitude of the P2 wave elicited by the first stimulus predicted the conscious detection of the second stimulus. This indicates that, during the time interval shortly preceding the second stimulus, at least some of the cortical generators of the P2 wave were more "responsive" to nociceptive input in those trials in which the second stimulus was perceived. Thus, it appears that the likelihood of perceiving the second stimulus was at least partly determined by spontaneous trial-to-trial fluctuations of the responsiveness of P2 cortical generators. What might be the functional significance of these trial-to-trial fluctuations? It is well known that a great part of the variability of any given cortical response can be attributed to the spontaneous dynamics of ongoing activity (Arieli et al., 1996; Fiser et al., 2004), and several studies have shown that the level of arousal is an important determinant of the magnitude of the P2 wave of LEPs (Beydoun et al., 1993; Legrain et al., 2002), as well as of stimulus detection and discrimination performance (Posner, 1980; Parasuraman, 1984). It is important to note that, although trial-to-trial fluctuations caused by mind wandering could account fully for the relationship between perception and the magnitude of the $\mathrm{P} 2$ wave elicited by the second stimulus, the same does not apply to the $\mathrm{N} 2$ wave. Indeed, perceiving or not perceiving the second stimulus conditioned the magnitude of the N2 wave elicited by the second stimulus, but not the magnitude of the N2 wave elicited by the first stimulus. This indicates that, distinct from the $\mathrm{P} 2$ wave, the $\mathrm{N} 2$ wave reflects processes that are related to the perceptual outcome of the stimulus, independently of spontaneous trial-to-trial fluctuations of the participant's attentional state.

Other studies have shown that the electrocortical activity preceding the onset of a stimulus (e.g., the magnitude of prestimulus alpha-band EEG oscillations) (Babiloni et al., 2006, 2008; Zhang et al., 2008) can predict sensory-discriminative performance as well as the magnitude of subsequent stimulus-evoked potentials. This relationship has also been hypothesized to reflect differences in the responsiveness of the cortical networks relevant to processing the stimulus.

Finally, it is important to note that the observed relationship between the magnitude of the N2 and P2 waves and the perceptual outcome of the nociceptive stimulus does not imply that these waves necessarily reflect cortical activity specific for the processing of nociceptive input (Mouraux and Iannetti, 2009).

\section{The effect of stimulus repetition}

The magnitudes of the N1, N2, and P2 waves were all significantly reduced by stimulus repetition (Fig. 5). Compared with the first stimulus, the second stimulus of the pair elicited N1, N2, and P2 waves of significantly smaller magnitude. The reduction in the magnitude of the EEG responses elicited by a repeated stimulus, often referred to as "repetition suppression" (Grill-Spector et al., 2006), occurs when identical stimuli are presented at a short (e.g., $<2$ s) and constant ISI (Hari et al., 1982; Angel et al., 1985; Raij et al., 2003; Iannetti et al., 2008). It has been conclusively demonstrated that this phenomenon is not attributable to neural refractoriness, but instead may result from the increased predictability of the repeated stimulus (Mouraux et al., 2004; Sable et al., 2004; Mouraux and Iannetti, 2008b). Indeed, when pairs of stimuli are presented at unpredictable ISIs, the magnitude of the response elicited by the second stimulus is entirely unaltered, even at intervals as short as $100 \mathrm{~ms}$ (Budd and Michie, 1994; Mouraux et al., 2004; Wang et al., 2008). In the present experiment, the effect of stimulus repetition affected equally the N1, N2, and P2 waves, and was present regardless of whether the second stimulus was perceived. This suggests that the neural mechanisms underlying repetition suppression do not require the conscious detection of the incoming sensory input, and would thus agree with the notion that repetition suppression reflects a preconscious mechanism preventing nonsalient sensory inputs from reaching consciousness (Sable et al., 2004).

\section{Conclusion}

Our results indicate that early-latency electrocortical responses to noxious stimulation (i.e., the $\mathrm{N} 1$ wave) reflect neural activity more related to the ascending nociceptive input, whereas later responses (i.e., the $\mathrm{N} 2$ and $\mathrm{P} 2$ waves) reflect functionally distinct neural activity more related to the emergence of pain from nociception.

This finding has a number of important implications, both in clinical practice and drug discovery. It indicates that the N1, N2, and P2 waves of LEPs can be used as functionally distinct biomarkers to explore neuropathic pain mechanisms (for example, the peripheral and central mechanisms thought to contribute to pain in fibromyalgia) (Lorenz et al., 1996). Furthermore, it indicates that LEPs can be used to characterize novel analgesics based on their modulation of nociceptive transmission and processing at peripheral and central levels.

\section{References}

Angel RW, Quick WM, Boylls CC, Weinrich M, Rodnitzky RL (1985) Decrement of somatosensory evoked potentials during repetitive stimulation. Electroencephalogr Clin Neurophysiol 60:335-342. 
Arieli A, Sterkin A, Grinvald A, Aertsen A (1996) Dynamics of ongoing activity: explanation of the large variability in evoked cortical responses. Science 273:1868-1871.

Babiloni C, Vecchio F, Bultrini A, Luca Romani G, Rossini PM (2006) Preand poststimulus alpha rhythms are related to conscious visual perception: a high-resolution EEG study. Cereb Cortex 16:1690-1700.

Babiloni C, Del Percio C, Brancucci A, Capotosto P, Le Pera D, Marzano N, Valeriani M, Romani GL, Arendt-Nielsen L, Rossini PM (2008) Prestimulus alpha power affects vertex N2-P2 potentials evoked by noxious stimuli. Brain Res Bull 75:581-590.

Baumgärtner U, Tiede W, Treede RD, Craig AD (2006) Laser-evoked potentials are graded and somatotopically organized anteroposteriorly in the operculoinsular cortex of anesthetized monkeys. J Neurophysiol 96:2802-2808.

Beydoun A, Morrow TJ, Shen JF, Casey KL (1993) Variability of laserevoked potentials: attention, arousal and lateralized differences. Electroencephalogr Clin Neurophysiol 88:173-181.

Bromm B, Treede RD (1984) Nerve fibre discharges, cerebral potentials and sensations induced by $\mathrm{CO}_{2}$ laser stimulation. Hum Neurobiol 3:33-40.

Budd TW, Michie PT (1994) Facilitation of the N1 peak of the auditory ERP at short stimulus intervals. Neuroreport 5:2513-2516.

Bushnell MC, Apkarian AV (2005) Representation of pain in the brain. In: Textbook of pain, Ed 5 (McMahon S, Koltzenburg M, eds), pp 267-289. Philadelphia: Churchill Livingstone.

Carmon A, Mor J, Goldberg J (1976) Evoked cerebral responses to noxious thermal stimuli in humans. Exp Brain Res 25:103-107.

Carmon A, Dotan Y, Sarne Y (1978) Correlation of subjective pain experience with cerebral evoked responses to noxious thermal stimulations. Exp Brain Res 33:445-453.

Craig AD (2009) How do you feel—now? The anterior insula and human awareness. Nat Rev Neurosci 10:59-70.

Fiser J, Chiu C, Weliky M (2004) Small modulation of ongoing cortical dynamics by sensory input during natural vision. Nature 431:573-578.

García-Larrea L, Peyron R, Laurent B, Mauguière F (1997) Association and dissociation between laser-evoked potentials and pain perception. Neuroreport 8:3785-3789.

García-Larrea L, Frot M, Valeriani M (2003) Brain generators of laserevoked potentials: from dipoles to functional significance. Neurophysiol Clin 33:279-292.

Giesbrecht B, Di Lollo V (1998) Beyond the attentional blink: visual masking by object substitution. J Exp Psychol Hum Percept Perform 24:1454-1466.

Grill-Spector K, Henson R, Martin A (2006) Repetition and the brain: neural models of stimulus-specific effects. Trends Cogn Sci 10:14-23.

Hari R, Kaila K, Katila T, Tuomisto T, Varpula T (1982) Interstimulus interval dependence of the auditory vertex response and its magnetic counterpart: implications for their neural generation. Electroencephalogr Clin Neurophysiol 54:561-569.

Iannetti GD, Truini A, Romaniello A, Galeotti F, Rizzo C, Manfredi M, Cruccu G (2003) Evidence of a specific spinal pathway for the sense of warmth in humans. J Neurophysiol 89:562-570.

Iannetti GD, Zambreanu L, Cruccu G, Tracey I (2005) Operculoinsular cortex encodes pain intensity at the earliest stages of cortical processing as indicated by amplitude of laser-evoked potentials in humans. Neuroscience 131:199-208.

Iannetti GD, Hughes NP, Lee MC, Mouraux A (2008) Determinants of laser-evoked EEG responses: pain perception or stimulus saliency? J Neurophysiol 100:815-828.

Jensen MP, Karoly P, O’Riordan EF, Bland F Jr, Burns RS (1989) The subjective experience of acute pain. An assessment of the utility of 10 indices. Clin J Pain 5:153-159.

Jung TP, Makeig S, Westerfield M, Townsend J, Courchesne E, Sejnowski TJ (2000) Removal of eye activity artifacts from visual event-related potentials in normal and clinical subjects. Clin Neurophysiol 111:1745-1758.

King AJ, Calvert GA (2001) Multisensory integration: perceptual grouping by eye and ear. Curr Biol 11:R322-R325.
Legrain V, Guérit JM, Bruyer R, Plaghki L (2002) Attentional modulation of the nociceptive processing into the human brain: selective spatial attention, probability of stimulus occurrence, and target detection effects on laser evoked potentials. Pain 99:21-39.

Lewis T, Ponchin EE (1937) The double pain response of the human skin to a single stimulus. Clin Sci 3:67-76.

Libet B, Alberts WW, Wright EW Jr, Feinstein B (1967) Responses of human somatosensory cortex to stimuli below threshold for conscious sensation. Science 158:1597-1600.

Loeser JD, Treede RD (2008) The Kyoto protocol of IASP Basic Pain Terminology. Pain 137:473-477.

Lorenz J, Grasedyck K, Bromm B (1996) Middle and long latency somatosensory evoked potentials after painful laser stimulation in patients with fibromyalgia syndrome. Electroencephalogr Clin Neurophysiol 100:165-168.

Meador KJ, Ray PG, Echauz JR, Loring DW, Vachtsevanos GJ (2002) Gamma coherence and conscious perception. Neurology 59:847-854.

Meredith MA, Nemitz JW, Stein BE (1987) Determinants of multisensory integration in superior colliculus neurons. I. Temporal factors. J Neurosci 7:3215-3229.

Mouraux A, Iannetti GD (2008a) Across-trial averaging of event-related EEG responses and beyond. Magn Reson Imaging 26:1041-1054.

Mouraux A, Iannetti GD (2008b) A review of the evidence against the "first come first served" hypothesis. Comment on Truini et al. [Pain 2007; 131:43-47]. Pain 136:219-221; author reply 222-223.

Mouraux A, Iannetti GD (2009) Nociceptive laser-evoked brain potentials do not reflect nociceptive-specific neural activity. J Neurophysiol 101:3258-3269.

Mouraux A, Guérit JM, Plaghki L (2003) Non-phase locked electroencephalogram (EEG) responses to $\mathrm{CO}_{2}$ laser skin stimulations may reflect central interactions between $\mathrm{A} \delta$ - and C-fibre afferent volleys. Clin Neurophysiol 114:710-722.

Mouraux A, Guérit JM, Plaghki L (2004) Refractoriness cannot explain why C-fiber laser-evoked brain potentials are recorded only if concomitant Adelta-fiber activation is avoided. Pain 112:16-26.

Nahra H, Plaghki L (2003) The effects of A-fiber pressure block on perception and neurophysiological correlates of brief non-painful and painful $\mathrm{CO}_{2}$ laser stimuli in humans. Eur J Pain 7:189-199.

Parasuraman R (1984) The psychobiology of sustained attention. In: Sustained attention in human performance (Warm JS, ed), pp 61-101. New York: Wiley.

Posner MI (1980) Orienting of attention. Q J Exp Psychol 32:3-25.

Raij TT, Vartiainen NV, Jousmäki V, Hari R (2003) Effects of interstimulus interval on cortical responses to painful laser stimulation. J Clin Neurophysiol 20:73-79.

Raymond JE, Shapiro KL, Arnell KM (1992) Temporary suppression of visual processing in an RSVP task: an attentional blink? J Exp Psychol Hum Percept Perform 18:849-860.

Sable JJ, Low KA, Maclin EL, Fabiani M, Gratton G (2004) Latent inhibition mediates N1 attenuation to repeating sounds. Psychophysiology 41:636-642.

Schubert R, Blankenburg F, Lemm S, Villringer A, Curio G (2006) Now you feel it-now you don't: ERP correlates of somatosensory awareness. Psychophysiology 43:31-40.

Treede RD (2003) Neurophysiological studies of pain pathways in peripheral and central nervous system disorders. J Neurol 250:1152-1161.

Treede RD, Meyer RA, Raja SN, Campbell JN (1995) Evidence for two different heat transduction mechanisms in nociceptive primary afferents innervating monkey skin. J Physiol 483:747-758.

Wang AL, Mouraux A, Liang M, Iannetti GD (2008) The enhancement of the N1 wave elicited by sensory stimuli presented at very short interstimulus intervals is a general feature across sensory systems. PLoS ONE 3:e3929.

Zhang Y, Wang X, Bressler SL, Chen Y, Ding M (2008) Prestimulus cortical activity is correlated with speed of visuomotor processing. J Cogn Neurosci 20:1915-1925. 